\title{
Crop Protection Compendium
}

Datasheet report for Zelus renardii (leafhopper assassin bug)

\section{Pictures}

\section{Picture Title Caption Copyright}

\section{Identity}

\section{Preferred Scientific Name}

Zelus renardii Kolenati

\section{Preferred Common Name}

leafhopper assassin bug

\section{EPPO code}

ZELURE (Zelus renardii)

\section{Taxonomic Tree}

\author{
Domain: Eukaryota \\ Kingdom: Metazoa \\ Phylum: Arthropoda \\ Subphylum: Uniramia \\ Class: Insecta \\ Order: Hemiptera \\ Suborder: Heteroptera \\ Family: Reduviidae \\ Genus: Zelus
}

Species: Zelus renardii 


\section{Distribution Table}

The distribution in this summary table is based on all the information available. When several references are cited, they may give conflicting information on the status. Further details may be available for individual references in the Distribution Table Details section which can be selected by going to Generate Report.

Last updated: 10 Jan 2020

\begin{tabular}{|c|c|c|c|c|c|c|c|}
\hline $\begin{array}{c}\text { Continent/Country } \\
\text { /Region }\end{array}$ & Distribution & $\begin{array}{c}\text { Last } \\
\text { Reported }\end{array}$ & Origin & $\begin{array}{c}\text { First } \\
\text { Reported }\end{array}$ & Invasive & Reference & Notes \\
\hline \multicolumn{8}{|l|}{ Asia } \\
\hline $\begin{array}{l}\text { Israel (/cpc/datasheet } \\
\text { /108457) }\end{array}$ & Present & & & 2018 & & CABI (Undated) & $\begin{array}{l}\text { Original citation: } \\
\text { van der Heyden } \\
(2018)\end{array}$ \\
\hline
\end{tabular}

\section{Europe}

\begin{tabular}{|c|c|c|c|c|}
\hline $\begin{array}{l}\text { Albania (/cpc/datasheet } \\
\text { /108354) }\end{array}$ & Present & 2016 & CABI (Undated) & $\begin{array}{l}\text { Original citation: } \\
\text { van der Heyden } \\
\text { (2017) }\end{array}$ \\
\hline $\begin{array}{l}\text { Greece (/cpc/datasheet } \\
\text { /108443) }\end{array}$ & Present & & CABI (Undated) & $\begin{array}{l}\text { Original citation: } \\
\text { van der Heyden } \\
(2015)\end{array}$ \\
\hline $\begin{array}{l}\text {-Crete (/cpc/datasheet } \\
\text { /108711) }\end{array}$ & Present & 2014 & CABI (Undated) & $\begin{array}{l}\text { Original citation: } \\
\text { van der Heyden } \\
(2015)\end{array}$ \\
\hline
\end{tabular}

\section{North America}

\begin{tabular}{|l|l|l|l|l|l|}
\hline $\begin{array}{l}\text { Canada (/cpc/datasheet } \\
\text { /108388) }\end{array}$ & Present & & & $\begin{array}{l}\text { CABI Data } \\
\text { Mining } \\
\text { (Undated) }\end{array}$ \\
\hline $\begin{array}{l}\text { United States } \\
\text { (/cpc/datasheet/108597) }\end{array}$ & Present & & & $\begin{array}{l}\text { CABI (Undated } \\
\text { a) }\end{array}$ \\
\hline $\begin{array}{l}\text {-Arizona (/cpc/datasheet } \\
\text { /108798) }\end{array}$ & Present & & $\begin{array}{l}\text { CABI Data } \\
\text { Mining } \\
\text { (Undated) }\end{array}$ & CABI (Undated \\
\hline $\begin{array}{l}\text {-California } \\
\text { (/cpc/datasheet/108799) }\end{array}$ & Present & & & a) \\
\hline $\begin{array}{l}\text {-Hawaii (/cpc/datasheet } \\
\text { /108806) }\end{array}$ & Present & & $\begin{array}{l}\text { CABI (Undated } \\
\text { a) }\end{array}$ \\
\hline
\end{tabular}




\section{Distribution Table Details}

Last updated: 10 Jan 2020

\begin{tabular}{|c|c|c|c|c|c|c|c|}
\hline $\begin{array}{c}\text { Continent/Country } \\
\text { /Region }\end{array}$ & Distribution & $\begin{array}{c}\text { Last } \\
\text { Reported }\end{array}$ & Origin & $\begin{array}{c}\text { First } \\
\text { Reported }\end{array}$ & Invasive & Reference & Notes \\
\hline
\end{tabular}

\section{Asia}

\begin{tabular}{|l|l|l|l|l|l|l}
\hline $\begin{array}{l}\text { Israel (/cpc/datasheet } \\
\text { /108457) }\end{array}$ & Present & & 2018 & CABI (Undated) $\begin{array}{l}\text { Original citation: } \\
\text { van der Heyden } \\
(2018)\end{array}$
\end{tabular}

\section{Europe}

\begin{tabular}{|c|c|c|c|c|}
\hline $\begin{array}{l}\text { Albania (/cpc/datasheet } \\
\text { /108354) }\end{array}$ & Present & 2016 & CABI (Undated) & $\begin{array}{l}\text { Original citation: } \\
\text { van der Heyden } \\
\text { (2017) }\end{array}$ \\
\hline $\begin{array}{l}\text { Greece (/cpc/datasheet } \\
\text { /108443) }\end{array}$ & Present & & CABI (Undated) & $\begin{array}{l}\text { Original citation: } \\
\text { van der Heyden } \\
(2015)\end{array}$ \\
\hline $\begin{array}{l}\text {-Crete (/cpc/datasheet } \\
\text { /108711) }\end{array}$ & Present & 2014 & CABI (Undated) & $\begin{array}{l}\text { Original citation: } \\
\text { van der Heyden } \\
(2015)\end{array}$ \\
\hline
\end{tabular}

\section{North America}

\begin{tabular}{|l|l|l|l|l|l|}
\hline $\begin{array}{l}\text { Canada (/cpc/datasheet } \\
\text { /108388) }\end{array}$ & Present & & & $\begin{array}{l}\text { CABI Data } \\
\text { Mining } \\
\text { (Undated) }\end{array}$ \\
\hline $\begin{array}{l}\text { United States } \\
\text { (/cpc/datasheet/108597) }\end{array}$ & Present & & & $\begin{array}{l}\text { CABI (Undated } \\
\text { a) }\end{array}$ \\
\hline $\begin{array}{l}\text {-Arizona (/cpc/datasheet } \\
\text { /108798) }\end{array}$ & Present & & $\begin{array}{l}\text { CABI Data } \\
\text { Mining } \\
\text { (Undated) }\end{array}$ & \\
\hline $\begin{array}{l}\text {-California } \\
\text { (/cpc/datasheet/108799) }\end{array}$ & Present & & & $\begin{array}{l}\text { CABI (Undated } \\
\text { a) }\end{array}$ \\
\hline $\begin{array}{l}\text {-Hawaii (/cpc/datasheet } \\
\text { /108806) }\end{array}$ & Present & & & $\begin{array}{l}\text { CABI (Undated } \\
\text { a) }\end{array}$ \\
\hline
\end{tabular}

\section{Natural enemy of}

\begin{tabular}{|l|l|l|l|}
\hline \multicolumn{1}{|c|}{ Species } & Stages attacked & Countries where known to occur & References \\
\hline Aphis gossypii (/cpc/datasheet/6204) & Adults/Nymphs & & \\
\hline Aphytis aonidiae (/cpc/datasheet/6607) & & \\
\hline Aphytis vandenboschi (/cpc/datasheet/6644) & & \\
\hline Bemisia tabaci (/cpc/datasheet/8927) & Adults/Nymphs & \\
\hline Drosophila melanogaster (/cpc/datasheet/19938) & & \\
\hline Glycaspis brimblecombei (/cpc/datasheet/25242) & Adults/Nymphs & \\
\hline Helicoverpa armigera (/cpc/datasheet/26757) & & \\
\hline Heliothis virescens (/cpc/datasheet/26774) & & \\
\hline Heteropsylla cubana (/cpc/datasheet/27919) & Adults/Nymphs & \\
\hline Pectinophora gossypiella (/cpc/datasheet/39417) & & \\
\hline Spodoptera exigua (/cpc/datasheet/29808) & & & \\
\hline
\end{tabular}




\section{References}

Davranoglou LR, 2011. Zelus renardii (Kolenati, 1856), a New World reduviid discovered in Europe (Hemiptera: Reduviidae: Harpactorinae). Entomologist's Monthly Magazine, 147(1766-68):157-162. http://www.pemberleybooks.com (/cpc/abstract/20113409335)

van der Heyden, T., 2015. A recent record of Zelus renardii (Kolenati, 1856) on Crete/Greece (Hemiptera: Heteroptera: Reduviidae: Harpactorinae). (Ein aktueller Nachweis von Zelus renardii (Kolenati, 1856) auf Kreta/Griechenland (Hemiptera: Heteroptera: Reduviidae: Harpactorinae)). BVnPC, 4(52), 55-59. https://www.biodiversidadvirtual.org /taxofoto/sites/default/files/ein_aktueller_nachweis_von_zelus_renardii_kolenati_1856_auf_kreta_griechenland.pdf (https://www.biodiversidadvirtual.org/taxofoto/sites/default/files /ein_aktueller_nachweis_von_zelus_renardii_kolenati_1856_auf_kreta_griechenland.pdf) van der Heyden, T., 2017. First records of Zelus renardii (Kolenati, 1856) (Hemiptera: Heteroptera: Reduviidae: Harpactorinae) for Albania. Arquivos Entomoloxicos, 18, 49-50. http://www.aegaweb.com/arquivos_entomoloxicos /ae18_2017_van_der_heyden_first_records_zelus_renardii_albania_hemiptera_reduviidae.pdf (http://www.aegaweb.com /arquivos_entomoloxicos/ae18_2017_van_der_heyden_first_records_zelus_renardii_albania_hemiptera_reduviidae.pdf) van der Heyden, T., 2018. First record of Zelus renardii Kolenati (Heteroptera: Reduviidae: Harpactorinae) in Israel. Revista Chilena de Entomología, 44(4), 463-465. https://biotaxa.org/rce/article/view/43714/37633 (https://biotaxa.org /rce/article/view/43714/37633)

\section{Distribution References}

CABI Data Mining, Undated. CAB Abstracts Data Mining.,

CABI, Undated. Compendium record. Wallingford, UK: CABI

CABI, Undated a. CABI Compendium: Status as determined by CABI editor. Wallingford, UK: CABI 


\section{World Map}

Analyzed by: Extent

Not recorded

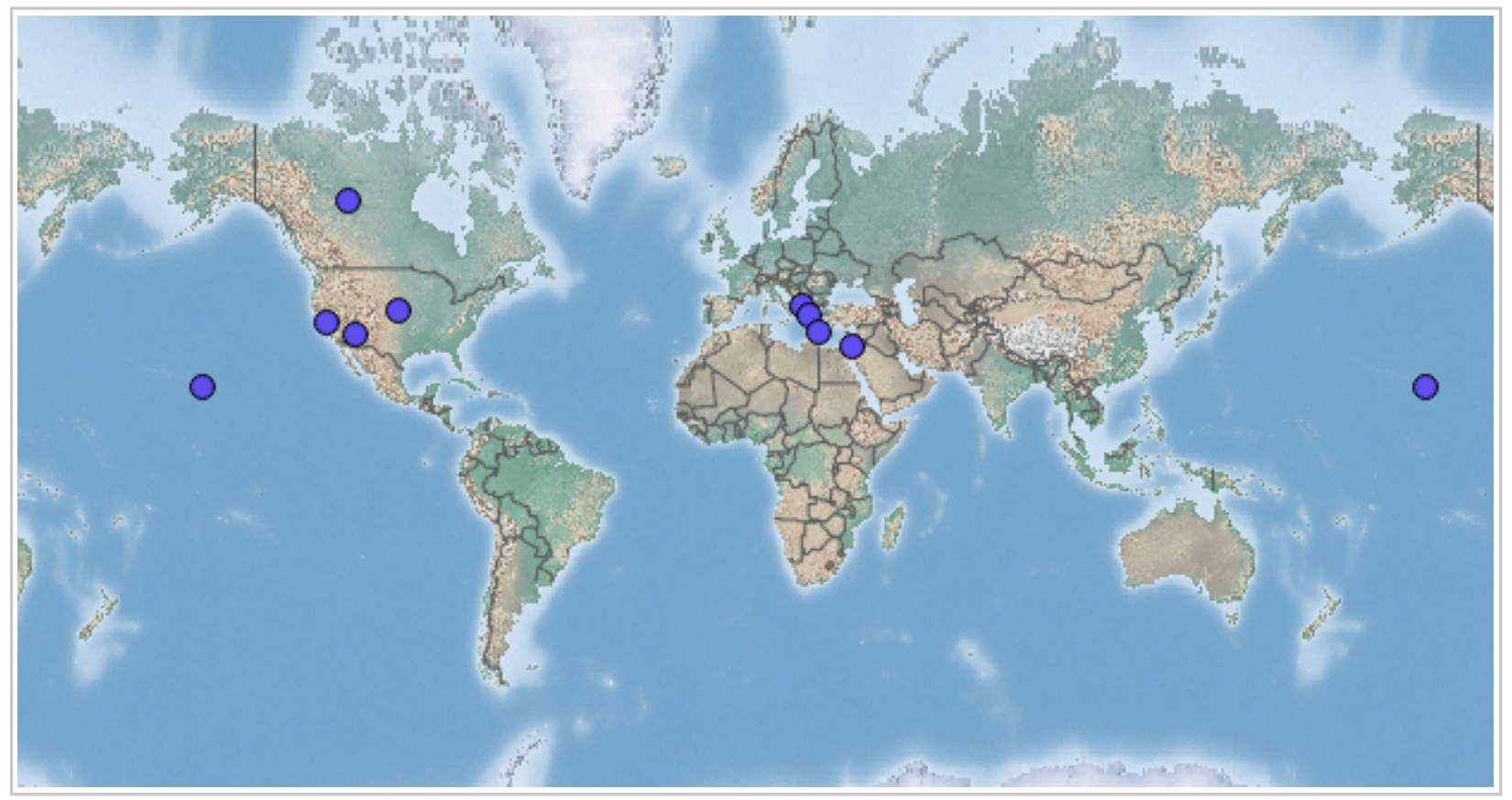




\section{Africa}

Analyzed by: Extent

Not recorded

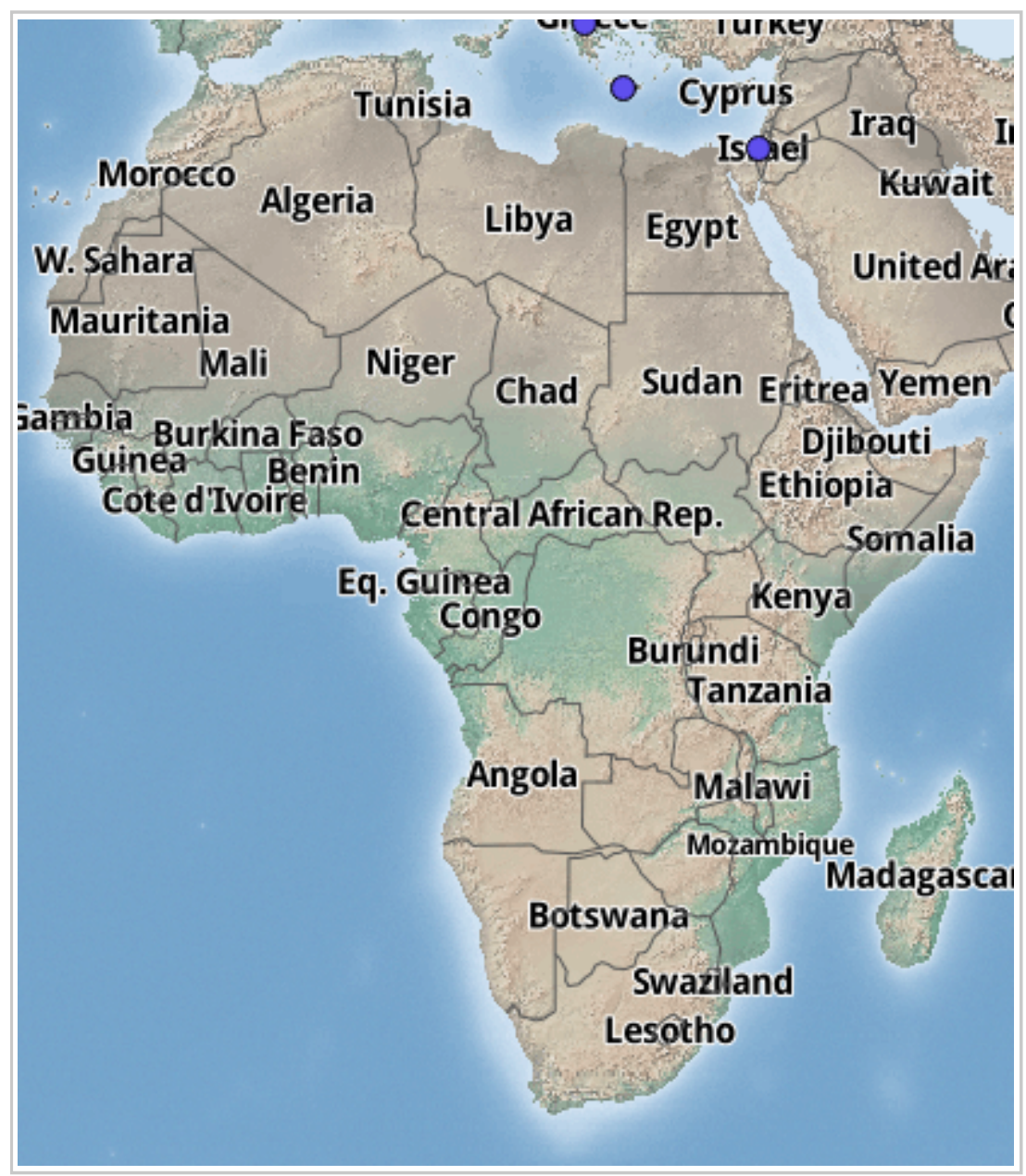




\section{Asia}

Analyzed by: Extent

Not recorded

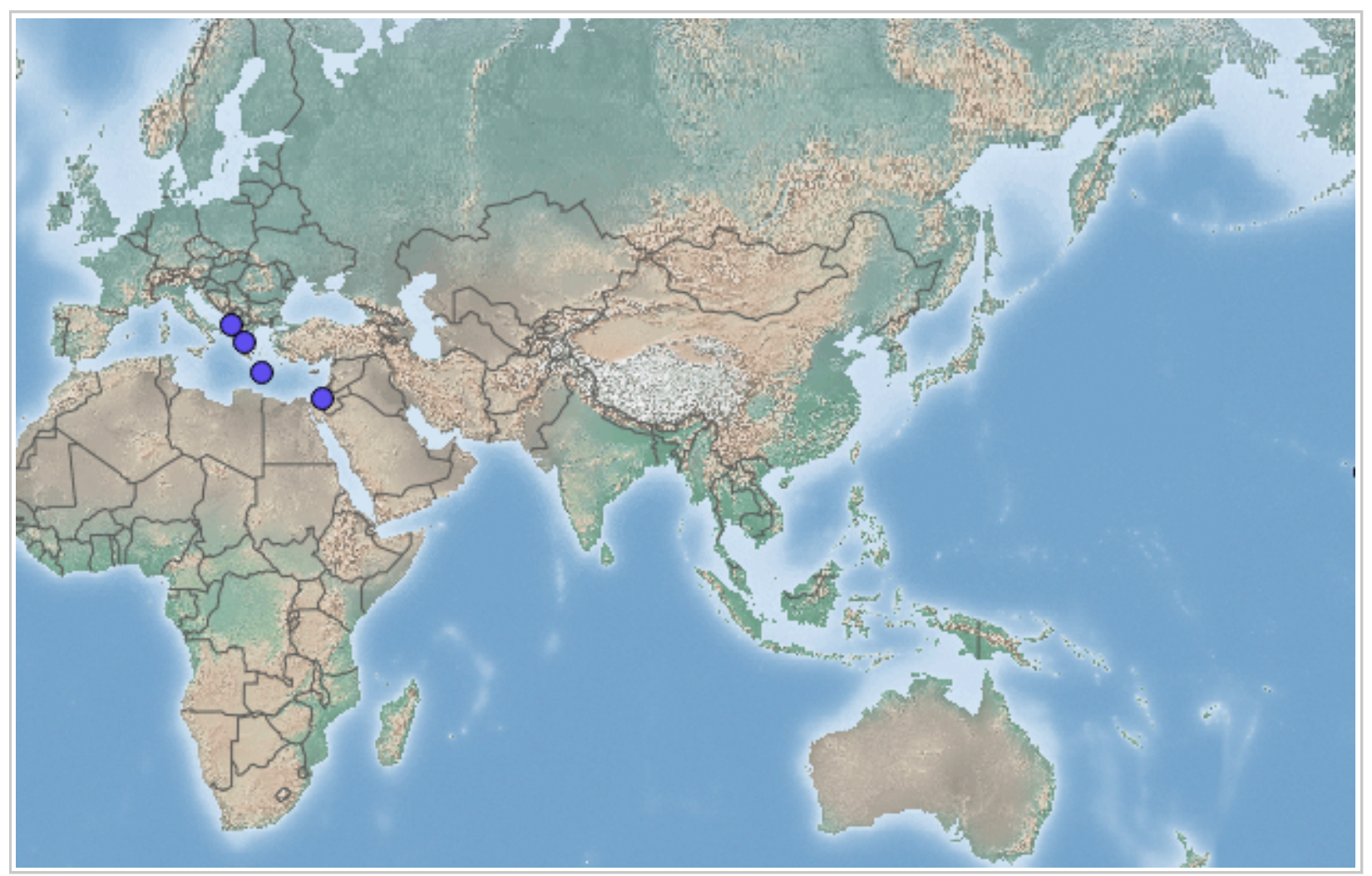




\section{Europe}

Analyzed by: Extent

Not recorded

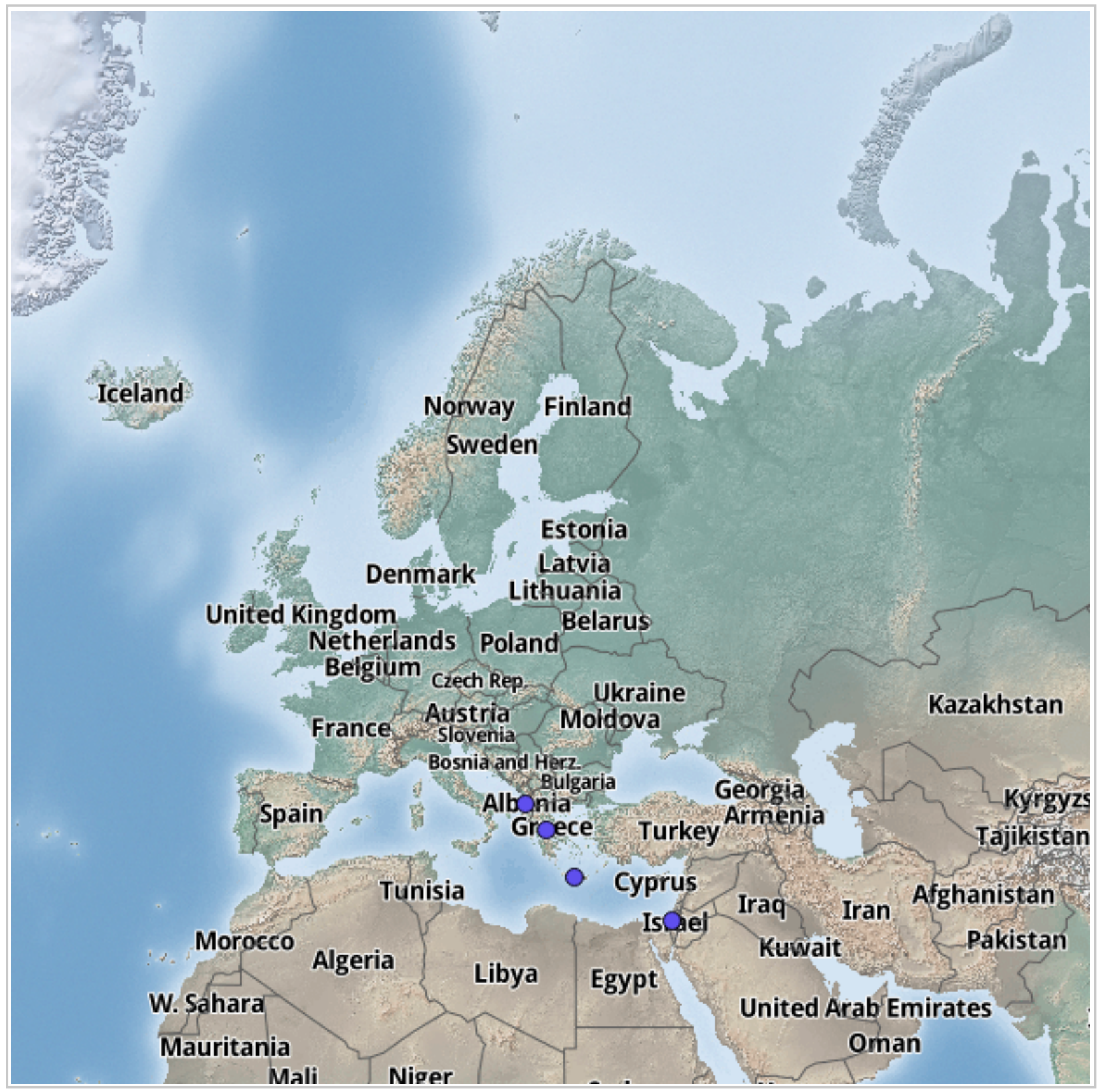




\section{Pacific}

Analyzed by: Extent

Not recorded

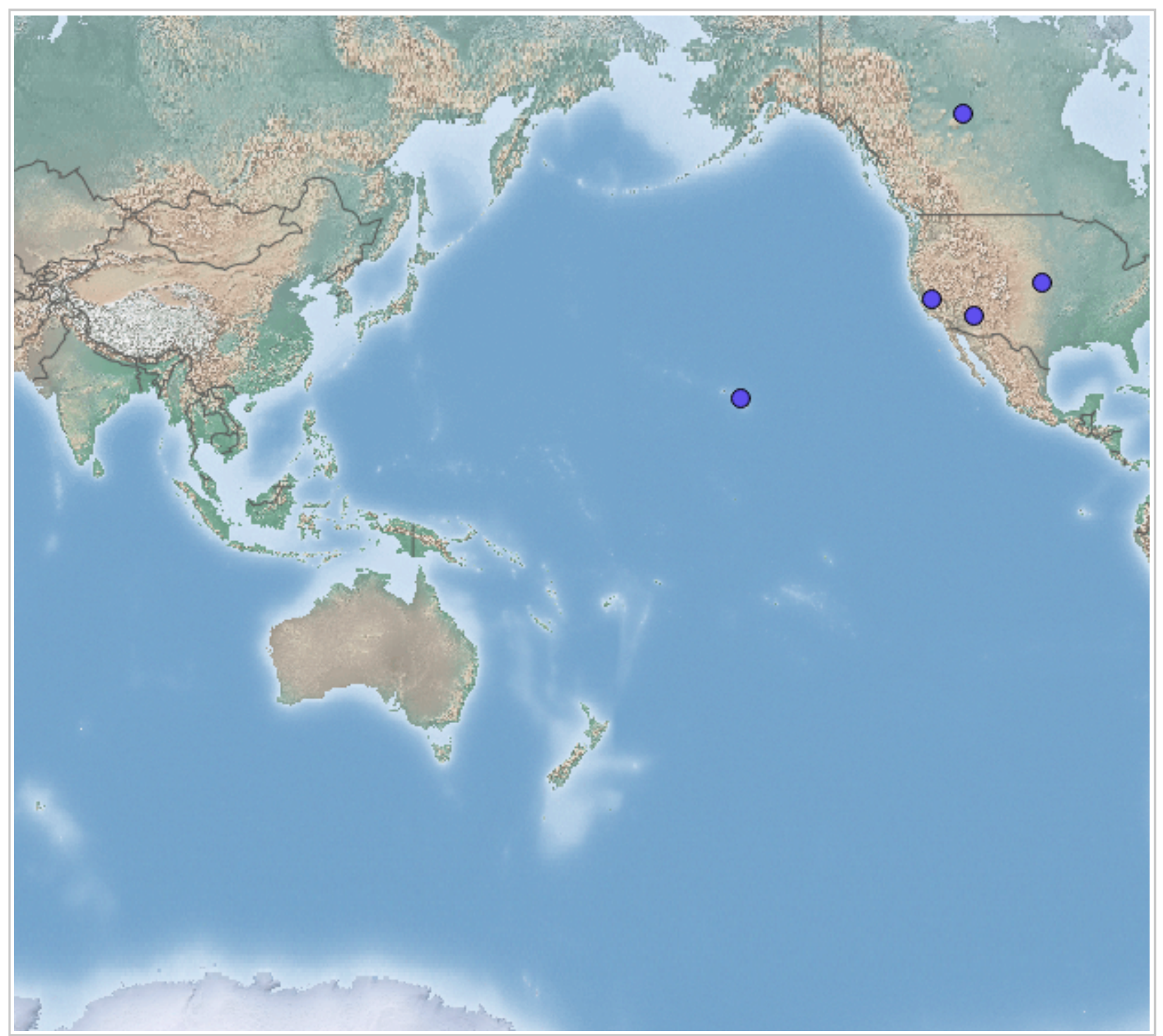




\section{North America}

Analyzed by: Extent

Not recorded

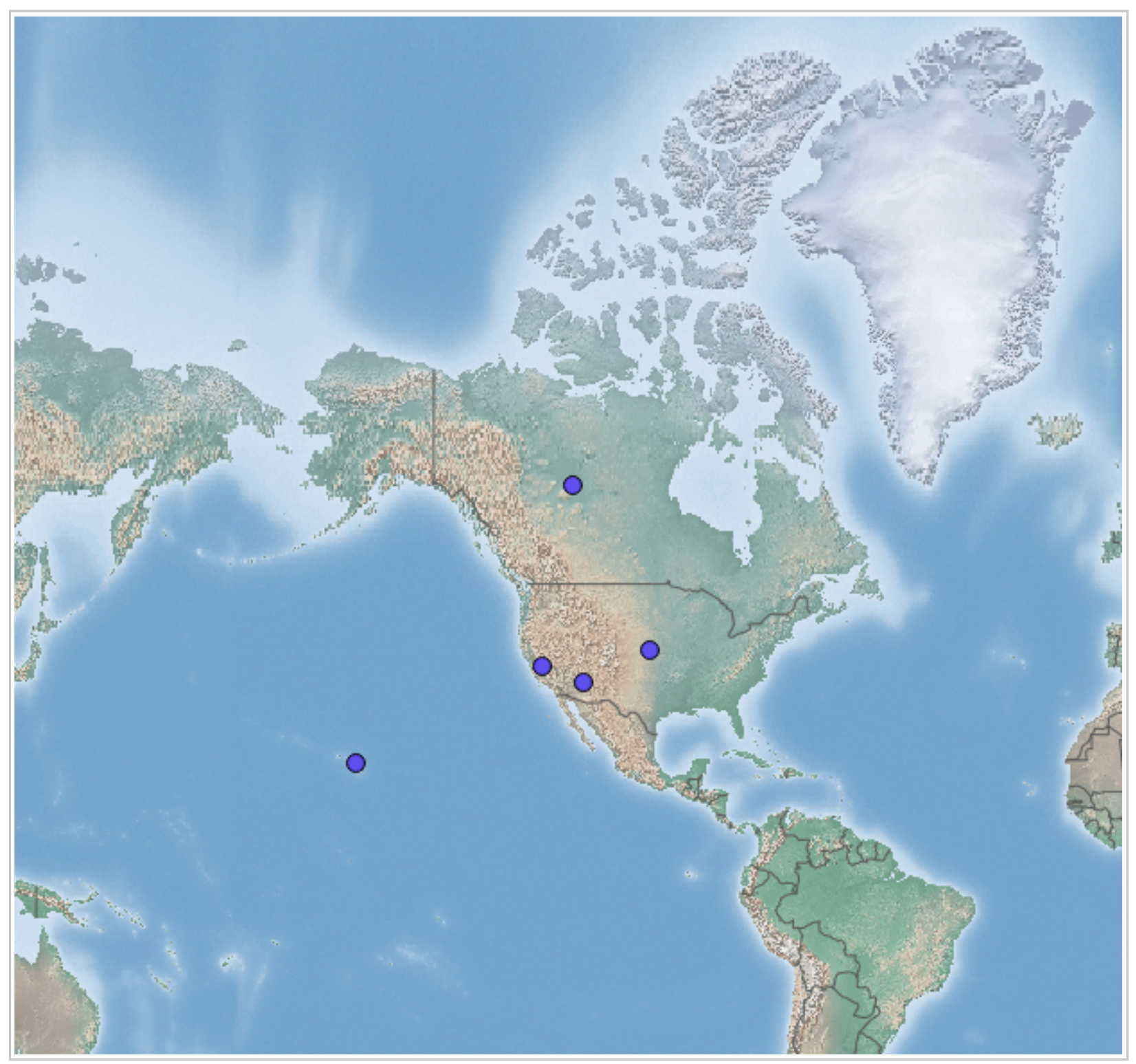




\section{Central America}

Analyzed by: Extent

Not recorded

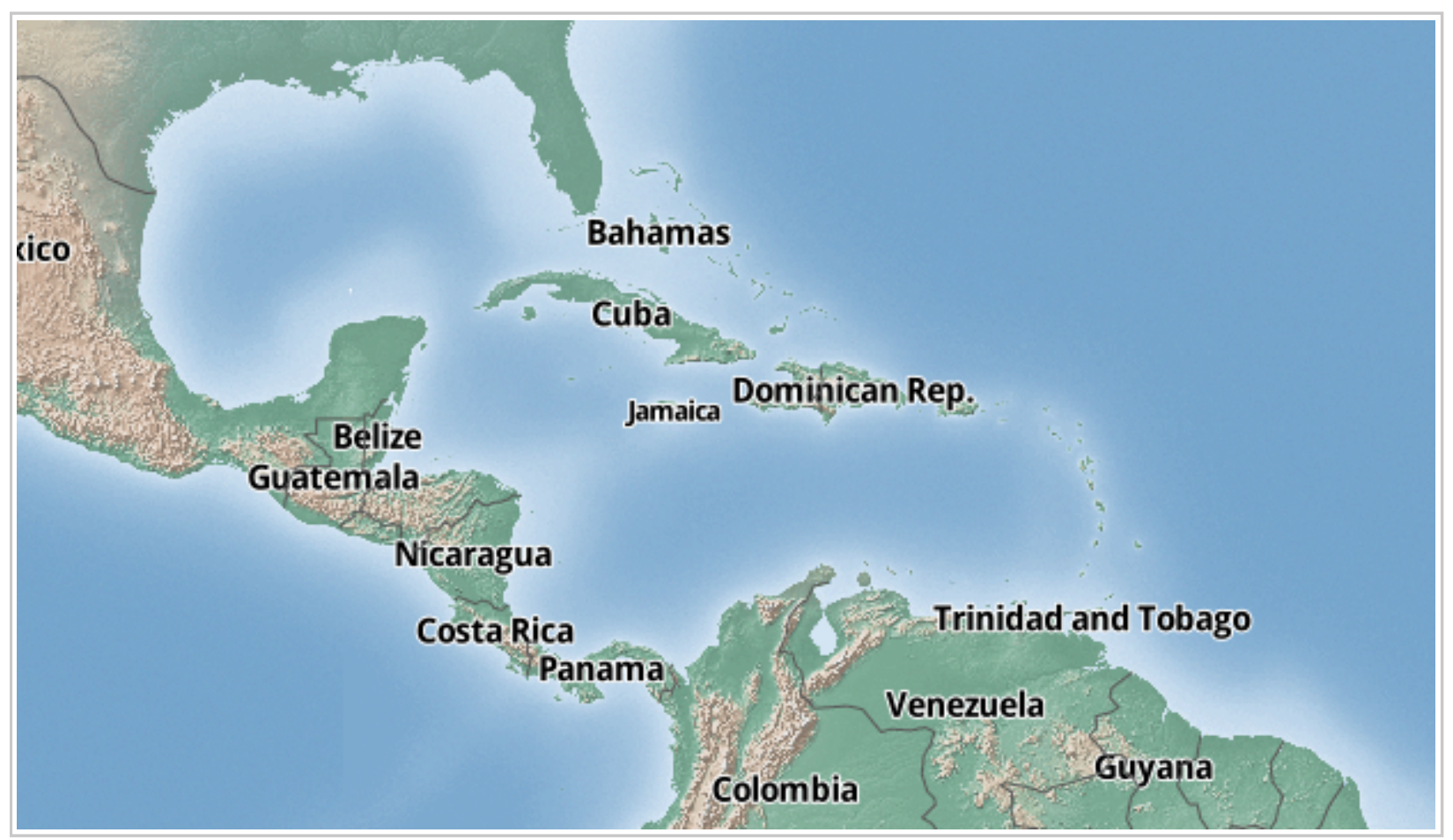




\section{South America}

Analyzed by: Extent

Not recorded

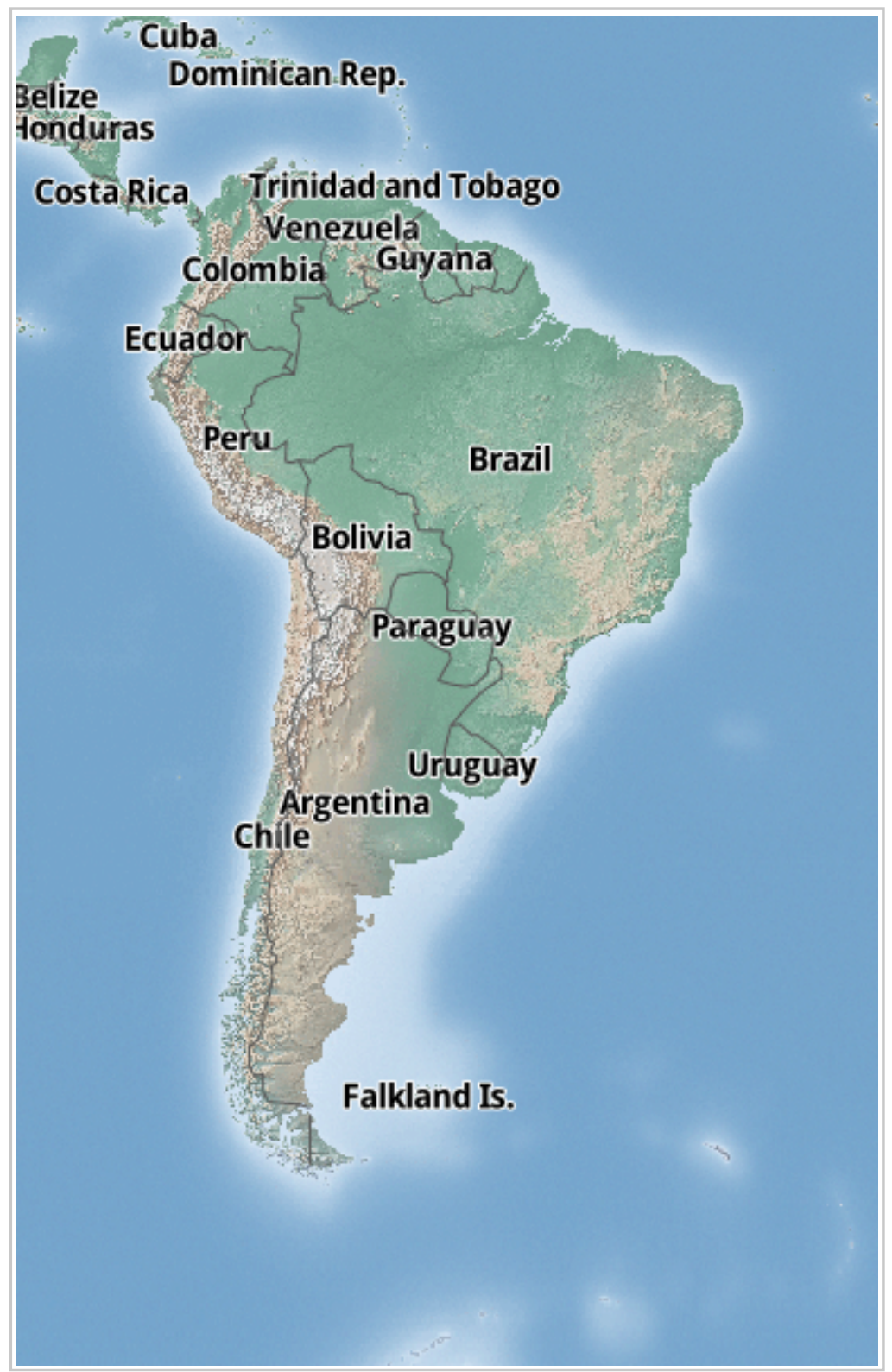

Date of report: 09 March, 2020 